\title{
High-Performance and Robust Strategy of Active Location Detection in WLAN
}

\author{
Siyu Zhan*, Xufa Wang, Jianming Liao, Jieyan Liu, Yalan Ye \\ University of Electronic Science and Technology of China, Chengdu, China. \\ * Corresponding author. Email: zhansy@uestc.edu.cn \\ Manuscript submitted January 25, 2016; accepted August 1, 2016. \\ doi: 10.17706/ijcce.2017.6.1.67-74
}

\begin{abstract}
The development of wireless network technologies gives attackers the convenience to use advanced radio technologies to hide their positions in wireless networks. While location service is a hot research currently, there are few location strategies concentrate on locating an attacker equipped with advanced radio technologies. In order to locate the attackers and make a security wireless network environment, we present a localization strategy, named as Active Location Detection (ALD), to locate attackers, without depending on the true features of attackers' signal. This process is robust against the attacks from these malicious users, using a finite horizon discrete Markov decision process (MDP). Furthermore, we qualitatively analyze the lower bound of ALD's error rate. After our analysis, a common formula of the lower bound of ALD's error rate is proved. Our simulations about ALD's error rate demonstrate that ALD's error rate is close to the lower bound of ALD's error rate. As further noted, the theories about the lower bound of ALD's error rate can also be applied to the range-free location systems.
\end{abstract}

Key words: Location, security, ALD, qualitative analysis, MDP.

\section{Introduction}

Although the wireless network technology provides a new and popular way to access networks, it also gives the attackers convenience to use advanced radio technologies, such as software defined radios (SDR) and smart antennas, to hide their positions in wireless networks. While location service is a hot research currently, there are a few location strategies concentrate on locating an attacker equipped with advanced radio technologies [1]-[3]. We propose a localization mechanism called Active Location Detection (ALD) for wireless networks to identify the real location of an attacker even if the attacker is equipped with advanced radio technologies.

There are two steps in the ALD process. In step one, the Packet Test Estimate (PTE) method is presented to calculate the upper bound of the distance between the attacker and its connecting access point. In the second step, we lure the attacker to change its signal features, force the attacker to reveal the true features of its signal, and locate the attacker. In order to get the estimated location quickly and reduce the probability that the attacker is alerted, the luring process of ALD is modeled as a finite horizon discrete Markov decision process (MDP).

After using the ALD process to identify an attacker's physical location in WLAN, a qualitative analysis of ALD's performance is also needed. So, we qualitatively analyze the lower bound of ALD's error rate. Extensive simulations about ALD's error rate demonstrate that ALD's error rate is close to the lower bound of ALD's error rate. 
The rest of the paper is organized as follows. Section 2 describes the related work. Section 3 introduce the ALD process. Its disassociation process is modeled as the finite horizon discrete MDP in Section 4. In Section 5, necessary qualitative analysis work about the lower bound of ALD's error rate is done. The simulated results are shown in Section 6. Final section concludes the whole paper.

\section{Related Work}

We analysis the challenges to locate an attacker equipped with SDR and smart antennas in GPS, range-free and range-based current location systems.

The GPS system has to trust the end device to report the correct location in the GPS system. Although tamper proof GPS receivers can be used to increase the reliability of location claims of end devices, which adds extra costs for legitimate users, it is impossible to enforce potential attackers to use tamper proof GPS devices. Range-free localization systems [4], [5] are designed to estimate a possible region of a target wireless node by collecting information about the node's connectivity with other nodes. These systems can be easily fooled by an attacker that intentionally distorts its signal information to prevent the systems to localize itself. Most range-based systems [6], [7] use absolute estimates of mutual distances or angles to locate a wireless node. Similar as in range-free systems, an attacker can fool range-based localization systems by distorting angle of arrival, power adaptation and transmission delay variation.

\section{The Process of ALD}

The process of ALD is divided into two steps: (a) Packet Test Estimate (PTE) method, deriving an upper bound of the distance between the attacker and its connecting AP. (b) Luring Connection (LC), luring the attacker to dynamically change its signal features.

In PTE, each AP has a capability to vary its transmission power level. After an attacker is connected to its home AP, none of the signal features can be directly used to localize the attacker. The upper bound derived by the PTE method is termed as PTE distance. To derive a tighter PTE distance, ALD controls the home AP to randomly use different transmission power levels while delivering packets to the attacker. By monitoring the lowest transmission power level that results in a successful response from the attacker, the home AP can derive a tight bound on the attacker's distance to itself. We call this bound as PTE distance.

The $L C$ process can actively lure the attacker to dynamically change its signal features, force the attacker to involuntarily reveal the true features of its signal, and then identifies its location. To narrow the location estimation coverage area down, ALD exploits the attacker's desire for communications to lure or force the attacker to change its home AP. When the attacker connects to a new home AP, a new PTE distance to this new home AP can be derived following the PTE method in step (a). Then, ALD obtains a new PTE estimation area centred at the new home AP. The location region of the attacker, hence, can be narrowed down to the intersection of the original estimation area and this new PTE estimation area. ALD repeatedly makes the attacker to switch its home AP until the targeted localization accuracy is reached or it is impossible to further narrow down the location estimation. When ALD ends its active localization effort, the attacker's real location area is revealed to be in the final location estimation region. In Section 4, we explain how to derive the optimal set of active APs at each $L C$ step.

\section{Markov Decision Process (MDP)}

To effectively locate an attacker, ALD must compute the best activation sequence of the attacker's neighbouring APs by considering the following three factors. First, we need to locate the attacker as fast as possible. Second, there is a risk that the attacker is alerted and moves to a new location in a LC step if the attacker is forced to connect to a new neighbouring AP but cannot see any APs. Hence, we associate with 
such an alert state with an alert "cost" factor $C_{\text {alert }}$ to capture the penalty caused by ALD's termination of its current localization process. Finally, in each LC step, the estimation region of the attacker's location is narrowed down. Hence, we define the "reward" in each LC step as the reduction of the attacker's location estimation area compared to the estimation in the previous LC step.

Considering these three factors, the best activation sequence of the LC steps should maximize the aggregated reward while minimizing the aggregated step cost and alert cost. To calculate this best trade-off point between reward and cost, ALD models the best activation sequence as a Markov decision process (MDP). Based on the above analysis, ALD finds the optimal activation sequence of APs by modeling this AP activation problem as a Markov decision process (MDP).

\subsection{Definition of MDP}

The MDP model for ALD's AP activation process is defined as a tuple $\left(S, A, A(s), P_{a}\left(s, s^{\prime}\right), r_{a}(s)\right)$ where $S$ is the state space, $A$ is the action space, $A(s)$ is the action space for state $s \in S, P_{a}\left(s, s^{\prime}\right)$ is the transition probability of a given action $a \in A(s)$ from state $s$ to state $s^{\prime}, r_{a}(s)$ is the expected immediate reward received after taking an action $a \in A(s)$ at state $s$. Each state $s_{X}$ in $S$ refers to the set of APs the attacker has connected to, where the subscript $x=x_{1}, x_{2}, \ldots, x_{k}$ represents that the attacker has connected to $x_{1}, x_{2}, \ldots$, and $x_{k}$. The estimation of the attacker's location region at state $s_{x}$, denoted as $\operatorname{Area}\left(s_{x}\right)$, is the intersection of the estimation disks of all APs in $x$. The only exception in the state space notation is the state that corresponds to the situations where the attacker is alerted. These states are represented by $s_{a l e r t}$.

\subsection{Calculation of MDP}

Given the cost and reward associated with each step of the operation, the goal of ALD's active-AP-selection operation is to maximize the aggregation of expected rewards minus costs by using an optimal sequence of activated APs. The optimal sequence can be computed as follows.

Given any state $s_{X}$, ALD determines the optimal set of activated APs, denoted by $\pi\left(s_{x}\right)$, as follows:

$$
\pi\left(\mathrm{s}_{\mathrm{x}}\right):=\arg \max _{\mathrm{a}}\left[r_{a}\left(s_{x}\right)+\gamma \sum_{y=x+z, z \in a} P_{a}\left(s_{x}, s_{y}\right) V_{s_{x}}\left(s_{y}\right)\right]
$$

where $V_{s_{x}}\left(s_{y}\right)$ represents the maximum expected aggregated reward from state $s_{x}$ to state $s_{y} . V_{s_{x}}\left(s_{y}\right)$ is defined as:

$$
V_{s_{x}}\left(s_{y}\right):=r_{\pi\left(s_{x}\right)}\left(s_{y}\right)+\gamma \sum_{z=y+n, n \in \pi\left(s_{y}\right)} P_{\pi\left(s_{y}\right)}\left(s_{y}, s_{x}\right) V_{s_{y}}\left(s_{z}\right)
$$

where $0<\gamma \leq 1$ is the discounting factor for the future reward and captures the fact that the future reward is less important due to the chance that the attacker may move in the future.

To find out the optimal AP activation process based on (1) and (2), there are two remaining problems, finding the transition probability between two states and defining the reward function.

\subsubsection{Expected transition probability calculation}

Given states $s_{x}, s_{y}$ and an action $a$, if the transition from $s_{x}$ to $s_{y}$ is possible, ALD calculates the transition probability based on the two assumptions. (a) At state $s_{x}$, it is assumed that the probability density of the adversary's location is uniform over the estimated region of state $s_{x}$. In this case, at any position in $s_{X}$, the attacker can connect to a subset of the active APs in the action $a$ and the attacker selects one in this subset as its new home AP. (b) We assume the selection of the attacker is completely random so that each active AP in this subset has an equal opportunity to be chosen. In other words, if the attacker is inside the intersection area of $n$ active APs in the subset, the attacker selects any one of them as its home AP with probability $1 / n$. 
With these assumptions, it can be proved the expectation transition probability from state $s_{x}$ to $s_{y}$ under action $n$ is:

$$
P_{a}\left(s_{x}, s_{y}\right)=\frac{1}{\operatorname{Area}\left(s_{x}\right)}\left[\sum_{v \in\left(2^{a}-\varnothing\right),(y-x) \in v} \frac{(-1)^{|v|-1}}{|v|} \operatorname{Area}\left(s_{x} \cap r_{v}\right)\right]
$$

where $\operatorname{Area}\left(s_{x}\right)$ is the area of the state $s_{x}$ and $r_{v}$ is the coverage region of AP $v$.

\subsubsection{Reward function calculation}

The reward function for an action $a$ at state $s$ is defined as a function of the decreased difficulty for locating the attacker within the region. Note that for a particular state, the larger is the estimated region of the attacker, the harder to locate the attacker within this region. Hence, the reward for transition from state $s_{x}$ to state $s_{y}$ can be represented by

$$
r\left(s_{x}, s_{y}\right)=\operatorname{Area}\left(s_{x}\right)-\operatorname{Area}\left(s_{y}\right)-C
$$

where $C$ is the constant cost for alert the attacker. Hence, the reward function in the MDP definition (1) becomes

$$
r_{a}\left(s_{x}\right)=\sum_{y=x+z, z \in a} P_{a}\left(s_{x}, s_{y}\right) r\left(s_{x}, s_{y}\right)
$$

The detailed proof of the formulas in MDP can be seen in our previous research [8].

\section{Qualitative Analysis of Lower Bound}

After proposing the ALD strategy to identify an attacker's physical location in WLAN, further qualitative analysis work about the lower bound of ALD's error rate is needed to judge the ALD's performance.

Definition 1: In a 2D located range $R$ with an area of $S$, APs' radio coverage radius $r$ can make the $R$ into $n$ disjoint located regions. The average localization error rate in the located range $R$ is:

$$
E_{\text {ave }}(R)=\sum_{i=1}^{n} p\left(R_{i}\right) e\left(R_{i}\right)
$$

where $p\left(R_{i}\right)$ is the probability of the located node in the range $R_{i}, e\left(R_{i}\right)$ is the average localization error rate in the range $R_{i}$. We assume that the node is uniformly distributed on the $2 \mathrm{D}$ closed region $R$, so

$$
p(x, y)=\left\{\begin{array}{l}
\frac{1}{S},(x, y) \in R \\
0, \text { otherwise }
\end{array}\right.
$$

where $p(x, y)$ is the probability of the located node in the coordinates $(x, y)$ point and $S$ is the area of the region $R$. Based on (7) , $E_{\text {ave }}(R)$ in (6) can be described as :

$$
E_{\text {ave }}(R)=\sum_{i=1}^{n} \frac{s_{i}}{S} \frac{1}{r_{i} s_{i}} \iint_{R_{i}} \sqrt{\left(x-x_{i}\right)^{2}+\left(y-y_{i}\right)^{2}} d x d y=\frac{1}{S r} \sum_{i=1}^{n} \iint \sqrt{\left(x-x_{i}\right)^{2}+\left(y-y_{i}\right)^{2}} d x d y
$$


where $s_{i}$ is the area of the located region $R_{i}, r_{i}$ is the radio coverage radius and $\left(x_{i}, y_{i}\right)$ is the barycenter coordinates for the located region $R_{i}$. Because $S$ and $r_{i}$ are all constants, we can transform (8) to

$$
E_{\text {ave }}(R)=C \sum_{i=1}^{n} W e\left(R_{i}\right)
$$

where $W e\left(R_{i}\right)=\iint_{R_{i}} \sqrt{\left(x-x_{i}\right)^{2}+\left(y-y_{i}\right)^{2}} d x d y$ and $C=1 / S r$.

Lemma 1: $W e\left(R_{i}\right)$ has the property of translation, which means in a given range $R_{i}$ the value of $W e\left(R_{i}\right)$ is nothing to do with the centroid position of $R_{i}$ in the coordinate system.

Here is the proof. If there is a located range $R$ and the initial position of the range's centroid is the origin of coordinates, the $W e(R)$ can be represented

$$
W e(R)=\iint_{R} \sqrt{x^{2}+y^{2}} d x d y
$$

If we translate $\mathrm{R}$ to a new range $D$, where $D=\left\{(u, v) \mid u=x-x_{R}, v=y-y_{R},(x, y) \in R\right\}$, then

$$
W e(R)=\iint_{R} \sqrt{x^{2}+y^{2}} d x d y=\iint_{D} \frac{\partial(x, y)}{\partial(u, v)} \sqrt{\left(x-x_{R}\right)^{2}+\left(y-y_{R}\right)^{2}} d u d v=\iint_{D} \sqrt{u^{2}+v^{2}} d u d v=W e(D)
$$

Lemma 2: There are two regions $R_{1}$ and $R_{2}$ in the 2D located and closed range $R . R_{1}$ and $R_{2}$ have the same shape and different area $s_{1}$ and $s_{2}$. It can be proved that:

$$
\frac{W e\left(R_{1}\right)}{W e\left(R_{2}\right)}=\left(\frac{s_{1}}{s_{2}}\right)^{\frac{3}{2}}
$$

where $R_{1}=\{(x, y) \mid(x, y) \in R\}, R_{2}=\{(u, v) \mid(u, v) \in R\}$.

Because of We( $\left.R_{1}\right)$ and We $\left(R_{2}\right)$ 's attribute of translation in Lemma 1, we can move the centroids of $R_{1}$ and $R_{2}$ to the origin of coordinates without changing the value of $W e\left(R_{1}\right)$ and $W e\left(R_{2}\right)$, then:

$$
\frac{W e\left(R_{1}\right)}{W e\left(R_{2}\right)}=\frac{\iint_{R_{1}} \sqrt{x^{2}+y^{2}} d x d y}{\iint_{R_{2}} \sqrt{u^{2}+v^{2}} d u d v}=\frac{\iint_{R_{2}} \frac{\partial(x, y)}{\partial(u, v)} \sqrt{\frac{s_{1}}{s_{2}} u^{2}+\frac{s_{1}}{s_{2}} v^{2}} d u d v}{\iint_{R_{2}} \sqrt{u^{2}+v^{2}} d u d v}=\left(\frac{s_{1}}{s_{2}}\right)^{\frac{3}{2}}
$$

Definition 2: We define $U w$ as the unit of $W e(R)$ and obtain $U w$ 's value by calculating $W e(R)$ 's value in a circle which area value is 1 :

$$
U w=\iint_{x^{2}+y^{2} \leq 1} \sqrt{x^{2}+y^{2}} d x d y=\frac{2}{3 \sqrt{\pi}}
$$

Definition 3: We define $A_{R}$ as the shape coefficient. $A_{R}$ 's value is the ratio between the $W e(R)$ corresponded in the unit area $U w$, when $S_{R}=1$, 


$$
A_{R}=\iint_{R} \sqrt{x^{2}+y^{2}} d x d y / U w
$$

It is easy to see that $A_{R} \geq 1$. We calculated some common shapes' $A_{R}$ value and the results are that: the circle is 1 , the hexagonal is 1.003 , the square AR is1.017.

According to (10), (11) and (12), $E_{\text {ave }}(R)$ in (9) can be written as:

$$
E_{\text {ave }}(R)=C \sum_{i=1}^{n} A_{i} s_{i}^{\frac{5}{2}} U w
$$

where $\forall 1 \leq i, j \leq n, \quad\left(R_{i} \cap R_{j}\right)=\varnothing, i \neq j$ and $\bigcup_{i=1}^{n} R_{i}=R$.

Theorem 1: In a 2D closed range $R$ with an area of $S$, where the attacker and the access points are uniformly distributed, the range $R$ can be divided into n disjoint regions by the APs' radio coverage radius $r$. With using the ALD localization algorithm, the average localization error rate's minimum value can be proved as $\frac{2 s}{3 r n} \sqrt{\frac{s}{\pi n}}$.

Proof: According to (13), $E_{\text {ave }}(R)$ can also be described as:

$$
E_{\text {ave }}(R)=C \sum_{i=1}^{n} A_{i} s_{i}^{\frac{5}{2}} U w \geq C U w \sum_{i=1}^{n} s_{i}^{\frac{5}{2}}
$$

If we make $g\left(s_{1}, s_{2},,, s_{n}\right)=\sum_{i=1}^{n} s_{i}^{\frac{5}{2}}\left(\sum_{i=1}^{n} s_{i}=S, s_{1},,, s_{n}>0\right)$ and use the method of Lagrange Multipliers, $g\left(g\left(s_{1}, s_{2},, s_{n}\right)\right.$ 's minimum value can be described as $\frac{S^{2}}{n} \sqrt{\frac{S}{n}}$. So we can transform the $E_{\text {ave }}(R)$ in (14) to

$$
E_{\text {ave }}(R) \geq C U w \sum_{i=1}^{n} s_{i}^{\frac{5}{2}}=\frac{1}{S r} \frac{2}{3 \sqrt{\pi}} \frac{S^{2}}{n} \sqrt{\frac{S}{n}}=\frac{2 S}{3 r n} \sqrt{\frac{S}{\pi n}}
$$

From the theorem above, we can conclude that: for the ALD algorithm, the higher the density of APs, the more number of areas divided in the plane, the less the average localization error rate. Furthermore, the theories about the lower bound of ALD's error rate can also be applied to the range-free location systems.

\section{Simulations}

We evaluate the performance of ALD in a grid topology where the AP density in the topology is represented by the ratio $r / d$, where $r$ is the radius of the coverage region of an AP and $d$ is the minimum distance between two APs. In our simulation, the discounting factor of ALD is set as $\gamma=0.9$. The location estimation of ALD is treated as the centroid of the estimated region. The coverage region of an AP is assumed to be a circle. In our simulations, we only consider the worst case. As to PTE distance in every step, we choose a random value between the attacker's realistic distance to AP and the $d$ because we assume we can't accurately measure the PTE distance by transmission power in worst case. As to the LC process, we still consider the worst case, which means we assume the attacker can be listened by only one AP in every step. In this simulation, we study the relationship between the APs density with the performance of ALD. 


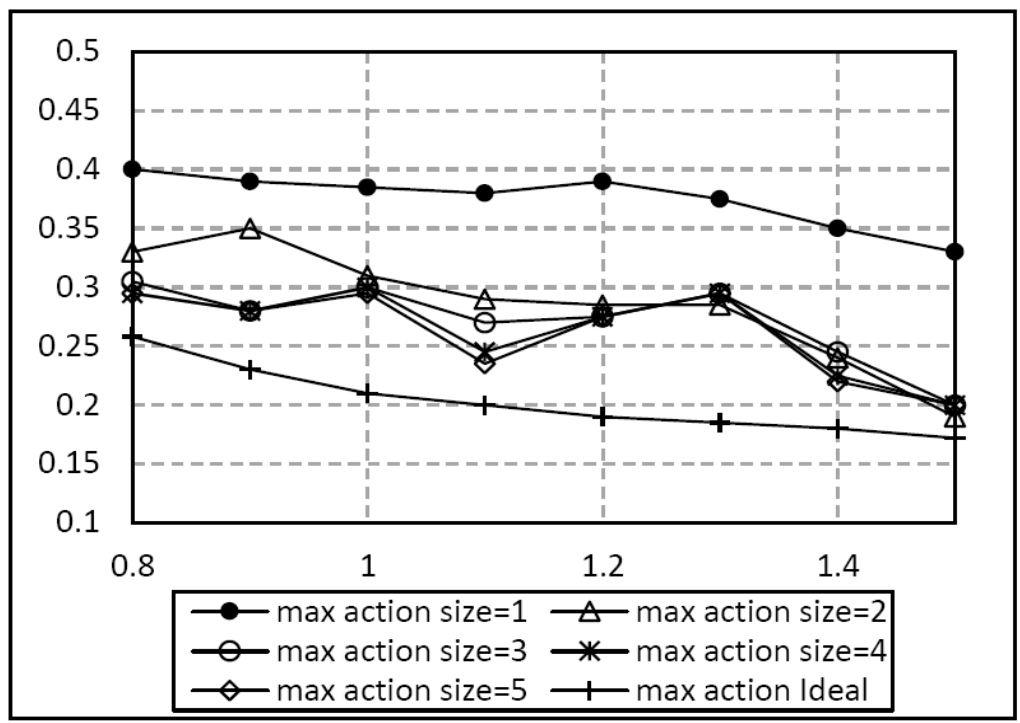

Fig. 1. ALD performance comparison.

As shown in the Fig. 1, the position error ratio decreases in a wave shape. When the network density is increased but the radio coverage radius is still not changed, the distance between access points will become smaller. Then, two neighboring access points' coverage area of intersection becomes larger, and the scope of the intersection area becomes larger that leads to the increase of the position error ratio. However, if the density continues to rise which will cause the access point intersects with an outer access point, the location area will shrink and the location error ratio will reduce.

In order to know the performance of ALD, we build mathematical models of ALD's error rate in Section 5. In the Fig. 1, we calculated the lower bounds in different AP densities by using the formula of the lower bound of ALD's error rate in Section 5. The lowest line in the Fig. 1 is the lower bound in different AP densities, named as "Ideal". We also find in the Fig. 1 that the ALD's error rate is close to the lower bound of ALD's error rate which visually proves ALD's high-performance.

\section{Conclusions}

In this paper, we present a range-free localization scheme, called ALD, to locate the attacker that initiatively hides its position information in WLAN. The ALD strategy does well in locating an attacker that distorts its signal features to hide its position. In order to judge the ALD strategy's performance, we build mathematical models of ALD's error rate and analysis the lower bound of ALD's error rate in Section 5. We also simulate some necessary scenarios to test and verify our theories about ALD's error rate.

\section{Acknowledgment}

This work is supported by the National Natural Science Foundation of China No. 61202444, No. 61202084, No. 61501096 and Chengdu Research Institute of UESTC No. RWS-CYHKF-02-20150005.

\section{References}

[1] Yingpei, Z., Jiannong, C., Jue, H., Shigeng, Z., \& Li, X. (2013). Secure localization and location verification in wireless sensor networks: A survey. Journal of Supercomputing, 64, 685-701.

[2] Wen, Z., Yang, X., Jianying, Z., Robert, D., \& Feng, B. (2011). Secure localization with attack detection in wireless sensor networks. International Journal of Information Security, 10, 155-171.

[3] Jeril, K., Sandeep, J., Vikram, R., \& Aravind, K. (2014). A review on localization in wireless sensor networks. In S. M. Thampi, A. Gelbukh and J. Mukhopadhyay (Eds.), Proceedings of Advances in 
Intelligent Systems and Computing: Vol. 264. Advances in Signal Processing and Intelligent Recognition Systems (pp. 599-610).

[4] Rania, K., Mohammed, E., \& Abdelhakim, M. (2015). Rang-free localization schemes for wireless sensor networks. TELKOMNIKA Indonesian Journal of Electrical Engineering, 16(2), 323-332.

[5] Jaehun, L., Wooyong, C., \& Euntai, K. (2011). A new range-free localization method using quadratic programming. Computer Communications, 34, 998-1010.

[6] Hetal, P. M., \& Nital, H. M. (2015). RSSI based localization scheme in wireless sensor networks: A survey. Proceedings of the Fifth International Conference on Advanced Computing \& Communication Technologies (ACCT) (pp. 647-652).

[7] Anthony, Q., Lars, K., \& Egemen, T. (2015). Combining range-based and range-free methods: A unified approach for localization. Proceedings of the 23rd SIGSPATIAL International Conference on Advances in Geographic Information Systems Article: Vol. 49.

[8] Zhan, S., Han, C., Yang, Y., \& Li, J. (2010). Active location detection of adversaries in 802.11 wireless LAN (WLAN). Chinese Journal of Electronics, 19(3), 525-531.

Siyu Zhan is an associate professor at the School of Computer Science and Engineering, University of Electronic Science and Technology of China (UESTC). He received the M.S. degree and Ph.D. degree in computer science from UESTC in 2005 and 2011 respectively. His research interests include wireless communications, networking and software engineering.

Xufa Wang is currently a master candidate at the School of Computer Science and Engineering, University of Electronic Science and Technology of China (UESTC).

Jianming Liao is a professor at the School of Computer Science and Engineering, University of Electronic Science and Technology of China (UESTC). He graduated in radio technology from Chongqing University in 1982. He is engaged in the research of computer networks, embedded systems and wireless sensor networks.

Jieyan Liu is currently a lecturer at the School of Computer Science and Engineering, University of Electronic Science and Technology of China (UESTC). She received M.S. degree and Ph.D. degree in computational applied science from UESTC in 2004 and 2012. She is interested in the research of computer network architecture.

Yalan Ye is an associate professor at the School of Computer Science and Engineering, University of Electronic Science and Technology of China (UESTC). She received Ph.D. degree in computer science from UESTC in 2008. Her research interests include networking and intelligent information processing. 